\title{
Diatoms in a future ocean - stirring it up
}

\section{Francesc Peters}

A recent review by Falkowski and Oliver ${ }^{1}$ argues that coccolithophorid algae, and possibly cyanobacteria and other picoplankton and nanoplankton, should dominate the upper mixed layer of the open ocean in the future. A scenario that involves increased ocean temperature would result in greater water-column stability and reduced turbulent mixing that, consequently, would reduce the input of inorganic nutrients from the deep ocean. This situation favours the growth of small plankton that have high surface to volume ratios over larger organisms, such as diatoms. Here, I contend that the presence of other factors renders this prediction of a generalized picoplankton and nanoplankton ocean uncertain.

Let me first consider the assumption that a future upper open ocean will become more stabilized. Air temperature is increasing to an even greater extent than ocean-water temperature ${ }^{2}$. Consequently, higher levels of water vapour in the atmosphere will more than likely increase storm frequency and intensity. Wind and storm events that temporarily increase turbulence in the upper mixed layer, and thus pulse nutrients into the euphotic zone, are the ideal growth conditions for diatoms. But, changes in the predominance of one type of 'life-form' (as defined by Margalef ${ }^{3}$ ) over an annual cycle are hard to predict, and the end result can be attributable to a trade off between the overall water-column stabilization and an increase in destabilization events. The uncertainty is large. Regionally, the global-warming trend is melting large areas of the Arctic, which is greatly reducing the summer ice-covered area; in summer 2007, the Arctic ice-free area was more than $10^{6} \mathrm{~km}^{2}$ larger than the previous record of 2005 (REF. 4).
This exposes large areas of the ocean to high wind-energy inputs that should favour organisms such as diatoms. In addition, the differential warming between the poles and the equator seems to intensify the westerlies ${ }^{5}$, entraining further mechanical energy into the upper ocean. It is clear that if trends other than that of water warming are considered, future oceanic conditions do not seem unfavourable for diatoms.

Let me now assume that the increase in upper-ocean temperature would indeed result in stronger thermal stratification and greater stability of the water column on average. The average nutrient availability from deep waters would be reduced, and our current tropical oceans are templates of this situation, with deep chlorophyll maxima (DCM) developing where enough nutrients meet sufficient radiation energy. Modelling shows that reduced vertical diffusivity in the water column results in a more fluctuating nutrient field near the DCM and an increase in the diversity of organisms ${ }^{6}$. This is because of mismatches between vertical settling rates of particulate organic matter and upward-flow velocities. It also explains the increase in diatom diversity with increasing temperature that has been observed in the geological record, as shown by Falkowski and Oliver ${ }^{1}$, and contradicts the conclusion that diatoms are 'losers' in such a situation.

In ocean areas of high nutrient, low chlorophyll (HNLC), export production after the pulsed addition of iron, which could represent an aeolian micronutrient input, is inversely related to the depth of the wind-mixed layer ${ }^{7}$. Diatoms take advantage of such pulsed conditions, and are also primarily responsible for export because of their heavy silicate frustules. Shoaling of the mixed layer in the vast HNLC areas, punctuated by micronutrient pulses, should then increase the export of organic matter to the deep ocean. This would reduce the excess atmospheric carbon dioxide, resulting in a negative feedback that would alleviate further temperature rises. Whether pulsing events or background stability would dominate the budget at annual scales and, consequently, the relative importance of diatoms, is again uncertain.

Thus, fluctuations or pulses that are caused by meteorological fronts, storms or aeolian inputs would be expected to increase in a global-warming scenario, and therefore have to be considered as drivers in the open ocean. Diversity should increase if such fluctuations can be considered as system disturbances of the right frequency ${ }^{8,9}$. Overall, future open-ocean scenarios do not point towards a decrease in the predominance of diatoms.

Francesc Peters is at the Institut de Ciències del Mar, CMIMA (CSIC), 08003 Barcelona, Catalunya, Spain. e-mail: cesc@icm.csic.es

Falkowski, P. J. \& Oliver, M. J. Mix and match: how climate selects phytoplankton. Nature Rev. Microbiol. 5, 813-819 (2007)

2. Solomon, S. et al. (eds) Climate Change 2007: the Physical Science Basis 1-996 (Cambridge Univ. Press, 2007)

3. Margalef, R. Life-forms of phytoplankton as survival alternatives in an unstable environment. Oceanol. Acta 1, 493-509 (1978).

4. Cressey, D. Arctic melts opens Northwest passage. Nature 449, 267 (2007).

5. Toggweiler, J. R. \& Russell, J. Ocean circulation in a warming climate. Nature 451, 286-288 (2008).

6. Huisman, J., Pham Thi, N. N., Karl, D. M. \& Sommeijer, B. Reduced mixing generates oscillations and chaos in the oceanic deep chlorophyll maximum. Nature 439, 322-325 (2006).

7. Boyd, P. W. et al. Mesoscale iron enrichment experiments 1993-2005: synthesis and future directions. Science 315, 612-617 (2007).

8. Sommer, U. An experimental test of the intermediate disturbance hypothesis using cultures of marine phytoplankton. Limnol. Oceanogr. 40, 1271-1277 (1995).

9. Connell, J. H. Diversity in tropical rain forests and coral reefs. Science 129, 1302-1310 (1978). 